\title{
Strong laser-pulse-driven ionization and Coulomb explosion of hydrocarbon molecules
}

\author{
Sergiy Bubin, Mackenzie Atkinson, and Kálmán Varga \\ Department of Physics and Astronomy, Vanderbilt University, Nashville, Tennessee 37235, USA \\ Xinhua Xie, Stefan Roither, Daniil Kartashov, Andrius Baltuška, and Markus Kitzler \\ Photonics Institute, Vienna University of Technology, A-1040 Vienna, Austria
}

(Received 23 March 2012; published 5 October 2012)

\begin{abstract}
Field ionization and Coulomb explosion of small hydrocarbon molecules driven by intense laser pulses are studied in a combined theoretical and experimental framework. The spectra of ejected protons calculated by the time-dependent density functional approach are in good agreement with the experimental data. The results of the simulations give detailed insight into the correlated electron and nuclear dynamics and complement the experiment with a time-dependent physical picture. It is demonstrated that the Coulomb explosion in the studied molecular systems is a sudden, all-at-once fragmentation where the ionization step is followed by a simultaneous ejection of the charged fragments.
\end{abstract}

DOI: 10.1103/PhysRevA.86.043407

PACS number(s): 33.80.Rv, 79.77.+g, 82.53.-k

With the advent of powerful light sources [1,2], the study of the interaction of strong short laser pulses with matter has become a focus of intense research interest. Various processes and phenomena have been investigated, including high-order harmonic generation [3], creation of attosecond pulses [4], control of molecular dissociation [5], ultrafast imaging [6], electron tunneling and diffraction [7], and Coulomb explosion $[8,9]$. Highly energetic dissociation of a molecule or solid due to multiple ionization, known as the Coulomb explosion, is a particularly interesting process because it exposes the key physical mechanisms associated with the electron and nuclear dynamics and ionization [9-13]. Once the laser strips electrons from the molecule, the remaining positively charged structure can explode, creating a molecular plasma cloud. Coulomb explosion can be used to generate bright $\mathrm{keV}$ x-ray photons [14,15], highly energetic electrons [16], and for imaging [17-19].

The strong-field ionization and fragmentation of hydrocarbon molecules is a prototypical example of the Coulomb explosion of polyatomic molecules, and it has been the subject of several experiments [9,11,20-23]. An important quantity measured in these experiments is the kinetic energy distribution of the protons ejected during the fragmentation. Proton energies in excess of $30 \mathrm{eV}$ at only very moderate peak intensities of the driving laser pulses have been reported for both large [11] and small hydrocarbon molecules [9]. The proton kinetic cutoff energies depend sensitively on the laser intensity and saturate at intensities that depend on the molecular species $[9,11]$. Earlier, this behavior was attributed to the creation of a long-lived charge localization state [11]. A more recent experiment [9], in contrast, suggested that the high kinetic proton energies originate from Coulomb explosions from a high molecular charge state. It was suggested [9] that a multibond version of the enhanced ionization process $[24,25]$ is responsible for reaching the observed high charge states, from which the protons are ejected simultaneously in a concerted Coulomb explosion process resulting in complete molecular fragmentations. A recent theoretical study [26] using a one-dimensional model of acetylene, $\mathrm{C}_{2} \mathrm{H}_{2}$, confirms the proposed ionization mechanism leading to the high charge states, but it did not investigate the fragmentation dynamics. While the experimental approaches allow the study of important aspects of the Coulomb explosion of molecules by analyzing the properties of the resulting fragments, they do not provide a complete, dynamical description of the internal mechanism taking place during the fragmentation.

In this work, the intense laser pulse induced electronic and nuclear dynamics, "the Coulomb explosion," of hydrocarbon molecules are studied theoretically in the framework of the time-dependent density functional theory (TDDFT) [27], and the results of the simulations are compared to experimental data. Two small molecules were investigated, namely methane, $\mathrm{CH}_{4}$, and 1,3-butadiene, $\mathrm{C}_{4} \mathrm{H}_{6}$. It is found that the Coulomb explosion of these hydrocarbon molecules results primarily from a sudden ionization followed by an all-at-once fragmentation, supporting a concerted Coulomb explosion picture. The intramolecular electron dynamics during and after the interaction with the laser pulses are simulated in the framework of real-space real-time TDDFT and complemented with Ehrenfest molecular dynamics for ionic motion. The TDDFT simulations are carried out using the adiabatic local density approximation (ALDA) with the parametrization of Perdew and Zunger [29]. Norm-conserving pseudopotentials [30] were used to represent core electrons. The initial state of the system was prepared by performing the ground-state DFT calculation. At the time-development stage, we used the following Kohn-Sham Hamiltonian:

$$
H=-\frac{\hbar^{2}}{2 m} \nabla_{\mathbf{r}}^{2}+V_{\mathrm{H}}(\mathbf{r}, t)+V_{\mathrm{XC}}[\rho](\mathbf{r}, t)+V_{\mathrm{ext}}(\mathbf{r}, t),
$$

where $\rho$ is the electronic density, defined as a sum over all occupied orbitals, $\rho(\mathbf{r}, t)=2 \sum_{k}\left|\psi_{k}(\mathbf{r}, t)\right|^{2} . V_{\mathrm{H}}$ and $V_{\mathrm{XC}}$ are the Hartree and exchange-correlation potential, respectively. Lastly, $V_{\text {ext }}$ is the external potential which includes the potential due to the moving ions, $V_{\text {ions }}$, and the potential due to the laser electric field in the dipole approximation, $V_{\text {laser }}=\mathbf{r} \cdot \mathbf{E}(t)$. The laser electric field was assumed as $\mathbf{E}(t)=$ $E_{\text {max }} \exp \left[-(1 / 2)\left(t-t_{\text {peak }}\right)^{2} / a^{2}\right] \sin (\omega t) \mathbf{e}_{x}$, polarized along $x$, with the parameters $a$ and $\omega$ matching those of the experiments, and the time when the intensity peaks was chosen to be 

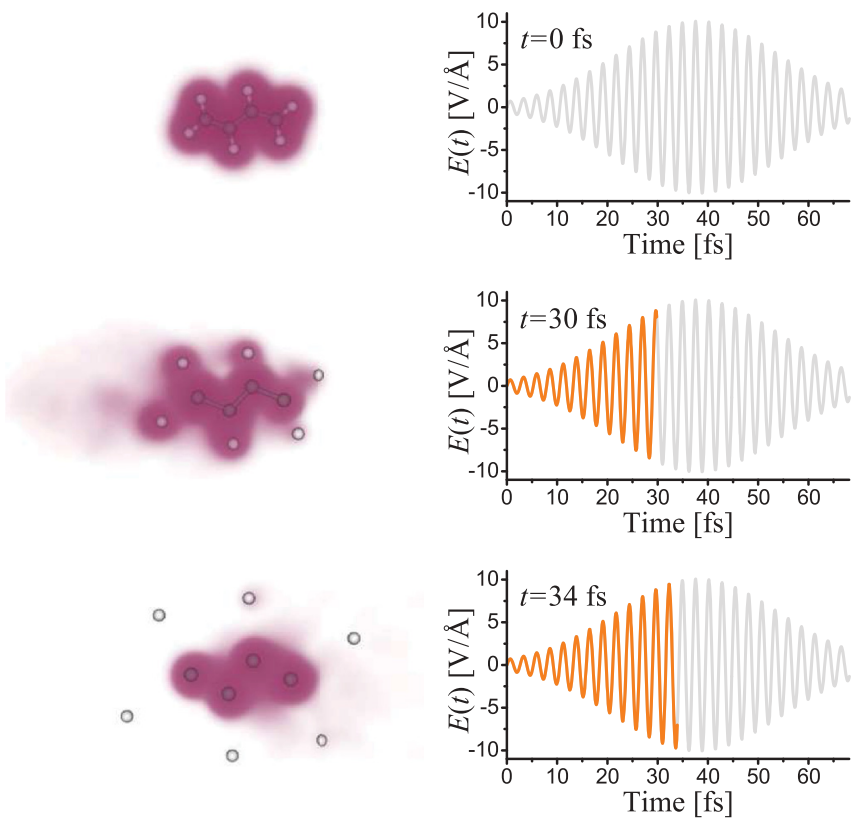

FIG. 1. (Color online) Snapshots of the 3D electron density and ionic positions of a $\mathrm{C}_{4} \mathrm{H}_{6}$ molecule subject to a laser pulse. For an animated version, see the supplemental material [28].

$t_{\text {peak }}=32.5 \mathrm{fs}$ after the start of the time propagation. For the $\mathrm{CH}_{4}$ molecule, we used two different laser peak intensities, $6.7 \times 10^{14}$ and $11.0 \times 10^{14} \mathrm{~W} / \mathrm{cm}^{2}$, and for the more computationally demanding simulations of $\mathrm{C}_{4} \mathrm{H}_{6}$, we limited ourselves to one intensity, $13.5 \times 10^{14} \mathrm{~W} / \mathrm{cm}^{2}$. Each KohnSham orbital, $\psi_{k}$, was time-propagated using the evolution operator in the form of the fourth-order Taylor expansion [31], with a small time step of 0.64 attoseconds. The total time window in our simulations was $80 \mathrm{fs}$, which we found to be sufficient to explore the dynamics and the mechanism of the proton ejection. All orbitals were represented on a realspace grid inside a large simulation volume $(37.75 \times 14 \times 14$ and $41.25 \times 17.5 \times 17.5 \AA$ for methane and 1,3-butadiene, respectively). The grid spacing was $0.25 \AA$. Initially, the molecules were placed at the center of the volume. To avoid unphysical reflection from the boundary of the simulation cell, we employed a complex absorbing potential (CAP) in a form similar to that proposed by Manolopoulos [32]. The CAP strength is zero within the central part of the simulation box and gradually reaches high magnitude near the $x$ sides.
In the experiment presented in this paper, coincidence momentum imaging is used to measure the three-dimensional momentum vector in the laboratory frame of each detected ion generated by ionization and fragmentation of methane, $\mathrm{CH}_{4}$, and 1,3-butadiene, $\mathrm{C}_{4} \mathrm{H}_{6}$, driven by the interaction with linearly polarized laser pulses of $27 \mathrm{fs}$ full width at halfmaximum (FWHM) duration carried at a center wavelength of $790 \mathrm{~nm}$. All other details of the experimental setup and data reconstruction procedure are as described previously [9].

Figure 1 shows snapshots of the valence electron density and ionic positions of a $\mathrm{C}_{4} \mathrm{H}_{6}$ molecule during the interaction with the laser pulse. At the leading edge of the laser pulse, the molecule becomes ionized. Then, the lighter ions (protons) are simultaneously ejected radially outward from the center of the molecule. At the last stage, the remaining carbon structure undergoes further ionization and explosion. For the simulation shown in Fig. 1, the protons are completely bare when they fly out of the system.

The process of ionization and fragmentation of a molecule in a laser electric field is strongly dependent on the orientation of the molecule with respect to the field polarization axis. In Fig. 1, the fragmentation process is visualized for a specific orientation of the $\mathrm{C}_{4} \mathrm{H}_{6}$ molecule, whereas in the experiments the molecules are randomly oriented. Thus, the measured proton spectra and charge states [9] are distributions coming from a large ensemble of systems with various spatial orientations. To model such distributions, we simulated 90 different orientations of each molecule that, by exploiting the molecular symmetry, cover all possible orientations in a randomly oriented ensemble. Figure 2 shows the evolution of ionization, i.e., the number of valence electrons in the system, of $\mathrm{CH}_{4}$ and $\mathrm{C}_{4} \mathrm{H}_{6}$ as a function of time. It can be seen that both investigated molecules become strongly ionized during the pulse, with molecular ionic charge states reaching the value $+5,+6$ in the case of $\mathrm{CH}_{4}\left(I=11.0 \times 10^{14} \mathrm{~W} / \mathrm{cm}^{2}\right)$ and +12 through +17 in the case of $\mathrm{C}_{4} \mathrm{H}_{6}$. The computed electron loss is in agreement with the experimentally determined values which have been extracted from the multiparticle coincidence data as described in Ref. [9]. Our simulations show that the molecules generally get more ionized when they are initially orientated such that there are more bonds parallel than perpendicular to the laser polarization. The different direction of the multiple bonds (both $\mathrm{C}-\mathrm{H}$ and $\mathrm{C}-\mathrm{C}$ ), however, makes it difficult to quantify a possible correlation between ionization yield and the orientation of the bonds.

The high degree of ionization leads to Coulomb explosion of the molecules. The dynamics of the ions, i.e., the dis-
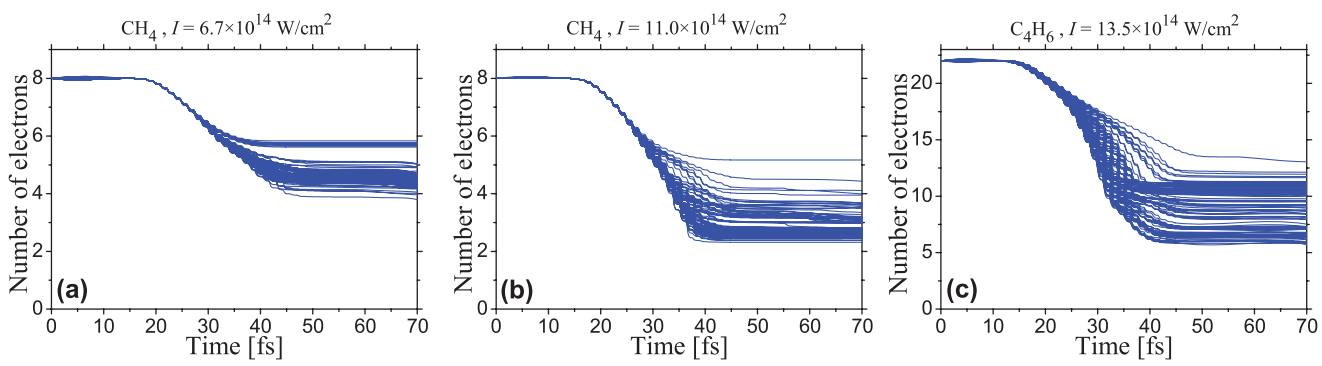

FIG. 2. (Color online) Number of valence electrons left in the system as a function of time for the entire ensemble of spatial configurations used in the calculations. 
placements from their initial positions, $\left|\mathbf{R}_{j}(t)-\mathbf{R}_{j}(0)\right|$, and the evolution of kinetic energies are illustrated in Figs. 3(c)-3(f). As can be seen, the molecules fragment completely and all protons get ejected simultaneously in a concerted process. The protons fly out in different directions as governed by the conservation of the total momentum but have very similar kinetic energies; see Figs. 3(e) and 3(f). While we only show the data for a single initial orientation of the $\mathrm{CH}_{4}$ and $\mathrm{C}_{4} \mathrm{H}_{6}$ molecules, the qualitative picture is the same for all other orientations used. Depending on a particular orientation, the final proton energies may vary substantially. Nonetheless, our simulations show that the kinetic energies of the individual protons ejected in the same explosion remain similar and the ejection occurs simultaneously for any given orientation. Therefore, both our simulations and experiment show that dynamic charge localization [11] does not apply to the fragmentation dynamics observed here, as a concerted emission of protons with similar energies is incompatible with the localization of the charge at a specific site within the molecule.

The fact that the protons are ejected concertedly is also reflected by the near zero kinetic energy of the remaining carbon ion of $\mathrm{CH}_{4}$ in both the simulated, Fig. 3(e), and measured data, Fig. 3(g). The measured carbon energy spectrum can be decomposed into the contributions of singly and doubly charged carbons (see the gray lines). Both of them peak at zero kinetic energy in accordance with a concerted, all-at-once emission of protons, which leaves the central carbon ion at rest. The similarity of the final kinetic energies of all ejected protons is most pronounced in the simulations with the highest laser intensity performed for the $\mathrm{C}_{4} \mathrm{H}_{6}$ molecule, Figs. 3(d) and 3(f). After the simultaneous departure of the protons, the heavy carbon skeletal structure separates in two steps, with each of them involving the explosion of two carbon ions, leading to two distinctly different final carbon energies. This dynamics is nicely reflected in the measured bimodal carbon energy spectrum in Fig. 3(h) with peaks at approximately 8 and $22 \mathrm{eV}$. A decomposition of the measured spectrum into the contributions of the singly and doubly charged carbon ions, shown by gray lines, reveals that the two peaks in the spectrum are caused by doubly and singly charged carbon ions, respectively. Figure 3(e) shows that for the slightly weaker laser peak intensity of $11.0 \times 10^{14} \mathrm{~W} / \mathrm{cm}^{2}$ used for the $\mathrm{CH}_{4}$ molecule, the high similarity of the proton energies observed for the high-intensity case of the 1,3-butadiene simulation becomes less pronounced. In the second simulation for $\mathrm{CH}_{4}$ with the still smaller intensity of $6.7 \times 10^{14} \mathrm{~W} / \mathrm{cm}^{2}$, the
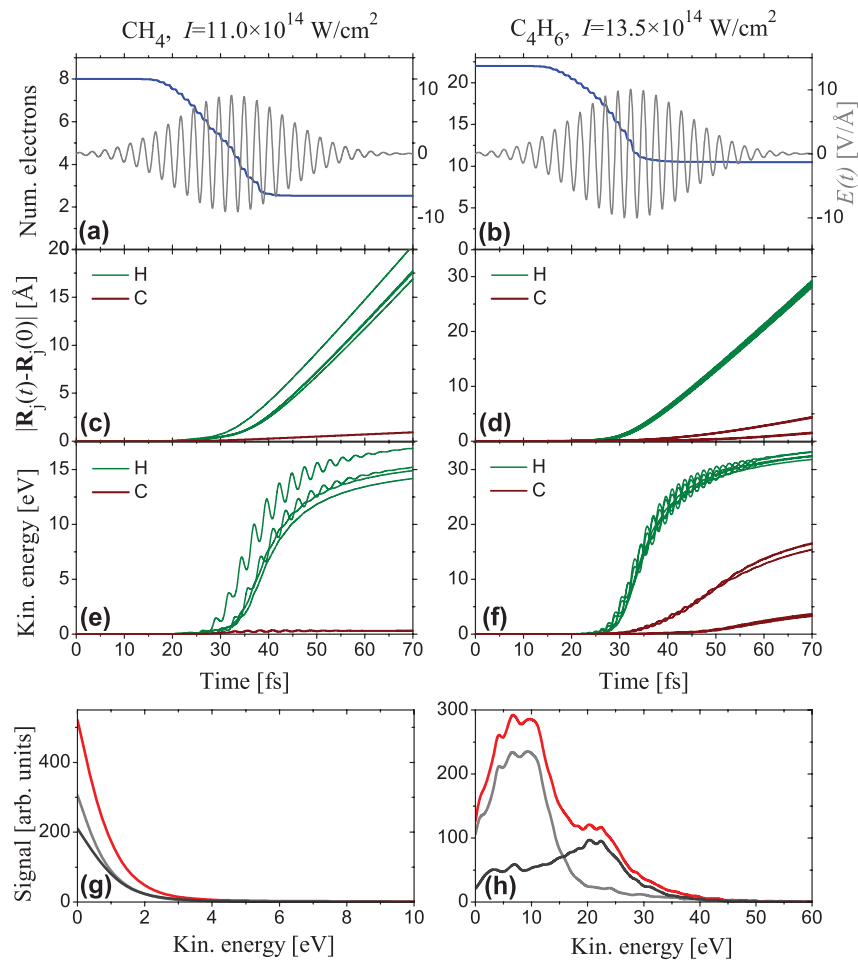

FIG. 3. (Color online) (a,b) Number of valence electrons remaining in the molecular system (blue lines) during ionization by a laser pulse (gray lines) for methane and 1,3-butadiene. (c,d) Displacements of individual protons (green lines) and carbon ions (dark red lines) from their initial positions. (e,f) Kinetic energies of individual protons (green lines) and carbon ions (dark red lines). While the data shown for the displacements and kinetic energies are for some randomly picked molecular spatial orientation, the same qualitative behavior could be observed for the majority of other orientations. (g,h) Measured carbon energy spectrum (red line) decomposed into the contributions of singly and doubly charged carbons (gray lines).

difference in the individual proton kinetic energies was quite noticeable, and for some of the initial spatial orientations the molecule did not undergo a complete fragmentation during the simulation time.

The total proton energy spectra that model the Coulomb explosion of $\mathrm{CH}_{4}$ and $\mathrm{C}_{4} \mathrm{H}_{6}$ are presented in Fig. 4. These spectra have been obtained as the histograms of the computed final energies of the protons. Upon complete molecular fragmentation, 90 different spatial configurations give rise to 360 and 540 ejected protons for methane and 1,3-butadiene, respectively,
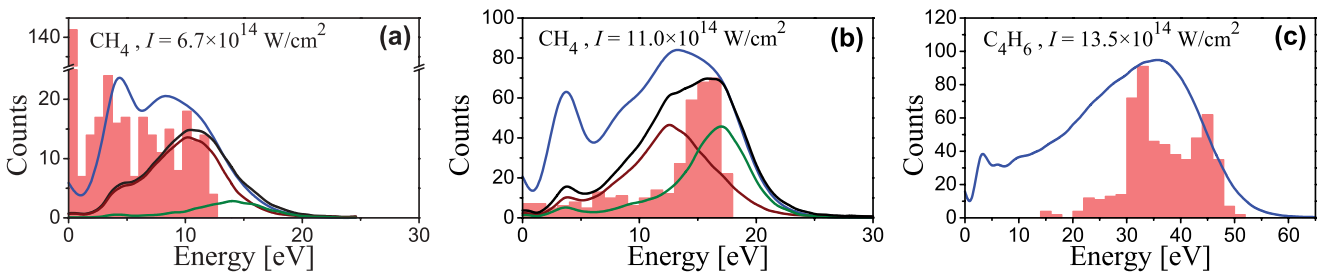

FIG. 4. (Color online) Histograms of computed proton energies for methane (a,b) and 1,3-butadiene (c), shown by the red bars, compared to experimentally obtained spectra (lines). The blue lines are the measured total proton energy spectra. For methane, these spectra are decomposed into the contribution coming from complete molecular fragmentations (black lines) resulting in a singly (red line) and doubly (green line) charged carbon ion. Laser peak intensities are indicated in the panels. 
which is sufficient for making a representative histogram of the proton spectra. We estimate the relative uncertainty of our final proton kinetic energies to be around 5\% of their values, with the uncertainties mainly due to finite simulation time and volume. In the lower intensity case for $\mathrm{CH}_{4}$, Fig. 4(a), one can observe a very tall first bin in the histogram. This peak is caused by those protons that for some spatial orientation of the molecule are not detached from the molecule within the 80 fs simulation period. The kinetic energies of all other protons are widely distributed up to the maximum value of $15 \mathrm{eV}$. The situation is very different when the peak intensity is increased to $11.0 \times$ $10^{14} \mathrm{~W} / \mathrm{cm}^{2}$; see Fig. 4(b). In that case, a pronounced peak at around 16-17 eV appears, while the number of protons ejected with low kinetic energies is relatively small, and the cutoff energy also increases to about $18 \mathrm{eV}$. The reason for the difference in the simulated proton energy spectra for low and high intensity can be understood by comparing them to measured spectra. The overall proton spectra (blue lines) consist in both intensity cases of a peak around $4 \mathrm{eV}$, which is due to protons ejected from doubly and triply charged methane, and a broad feature at higher energy. By selecting only those protons that are created by complete fragmentations and the production of a singly or doubly charged carbon ion [9], see Figs. 4(a) and 4(b), it becomes clear that the many low-energy protons in the simulation with the lower laser peak intensity, Fig. 4(a), are created by incomplete molecular fragmentations. The protons with high energy are created only if the molecules fragment completely.

In contrast, for the higher laser intensity used for simulating the spectrum in Fig. 4(b), almost all protons are created by complete molecular fragmentations, and the position of the pronounced high-energy feature observed in the simulation agrees well with measured protons correlated to singly and doubly charged atomic carbon ions created during complete molecular decomposition. We attribute the remaining small but discernible difference in the cutoff energy of the simulated and measured spectra to the uncertainty in determining absolute values for the experimental pulse peak intensity. As the ionization rate is highly nonlinear in intensity, even small uncertainties in the few $10 \%$ range may result in noticeable variations of the cutoff energy, in particular for smaller laser intensities.

We have shown that the protons that appear in the highenergy region of the spectrum are created by complete fragmentations only. Clearly, part of the low-energy experimental proton spectra are due to the spatial intensity distribution across the focused laser beam. Molecules that are ionized in the wings of this distribution are effectively exposed to a lower intensity than that assumed in the simulation. This effect causes part of the deviation in the low-energy region between the measured overall proton spectrum and the decomposed spectra that correspond to complete molecular fragmentations. However, this intensity smearing is absent in the simulated spectra. Thus, the broad proton energy distribution in the simulated spectrum extending to the low-energy region [Fig. 4(a)] is a signature that the high charge states, necessary for complete fragmentation and high-energy proton ejection, are not reached for all molecular orientations. As a consequence, the underlying ionization mechanism is strongly dependent on the orientation of the molecules relative to the laser polarization direction, motivating further experiments on aligned molecules [7,33,34].

Finally, we turn to the case of the highest intensity used in the simulation for the $\mathrm{C}_{4} \mathrm{H}_{6}$ molecule; see Fig. 4(c). The simulated spectrum does not show any low-energy protons but features a bimodal distribution centered around $40 \mathrm{eV}$. This means that the probability for complete molecular fragmentation from high charge states of 1,3-butadiene at this intensity is roughly $100 \%$, independent of the molecular orientation. The high-energy region of the measured proton energy spectrum agrees very well with the simulated spectrum. Although we are unable to decompose the measured spectrum into the contributions of the separate fragmentation channels, because their corresponding carbon momentum spectra overlap for carbon charge states higher than two [9], a comparison with the simulated spectrum allows us to attribute the lower-energy protons in the measured spectrum to incomplete fragmentations that occur at regions of smaller intensity within the spatial laser beam profile. The simulations show that at this intensity, the number of electrons being detached from 1,3-butadiene during the laser pulse peaks around 15-17 and 11-12 for the complete ensemble of 90 molecular orientations, as can be seen in Fig. 2(c). Thus, the two peaks of the bimodal proton spectrum can be attributed to the existence of two strong fragmentation channels from different ionic charge states.

In summary, we have investigated the electron and ion dynamics during laser-driven Coulomb explosion of small hydrocarbon molecules using real-space real-time TDDFT. The results of the simulations agree fairly well with measured data. We have shown that, in agreement with the scenario proposed previously [9], protons are ejected from the same molecular ion with no delay between any of the individual ejections, provided the laser pulse intensity is high enough to cause a complete fragmentation of the molecule. We have also shown that the kinetic energies of the individual protons ejected in the same explosion are very similar, and the wide distributions seen in the the experimental spectra result from different spatial orientations of molecules in the gas phase.

This work has been supported by the National Science Foundation (Grant No. CMMI-0927345) and by the Austrian Science Fund (FWF) (Grants No. P21463-N22 and No. I274N16).
[1] M. Protopapas, C. H. Keitel, and P. L. Knight, Rep. Prog. Phys. 60, 389 (1997).

[2] G. A. Mourou, C. P. J. Barry, and M. D. Perry, Phys. Today 51(1), 22 (1998).

[3] Z. Chang, A. Rundquist, H. Wang, M. M. Murnane, and H. C. Kapteyn, Phys. Rev. Lett. 79, 2967 (1997).
[4] M. Hentschel, R. Kienberger, C. Spielmann, G. A. Reider, N. Milosevic, T. Brabec, P. Corkum, U. Heinzmann, M. Drescher, and F. Krausz, Nature (London) 414, 509 (2001).

[5] R. J. Levis, G. M. Menkir, and H. Rabitz, Science 292, 709 (2001). 
[6] W. Li, X. Zhou, R. Lock, S. Patchkovskii, A. Stolow, H. C. Kapteyn, and M. M. Murnane, Science 322, 1207 (2008).

[7] M. Meckel, D. Comtois, D. Zeidler, A. Staudte, D. Pavičić, H. C. Bandulet, H. Pépin, J. C. Kieffer, R. Dörner, D. M. Villeneuve, and P. B. Corkum, Science 320, 1478 (2008).

[8] T. Ditmire, J. W. G. Tisch, E. Springate, M. B. Mason, N. Hay, R. A. Smith, J. Marangos, and M. H. R. Hutchinson, Nature (London) 386, 54 (1997).

[9] S. Roither, X. Xie, D. Kartashov, L. Zhang, M. Schöffler, H. Xu, A. Iwasaki, T. Okino, K. Yamanouchi, A. Baltuska, and M. Kitzler, Phys. Rev. Lett. 106, 163001 (2011).

[10] S. Palaniyappan, R. Mitchell, R. Sauer, I. Ghebregziabher, S. L. White, M. F. Decamp, and B. C. Walker, Phys. Rev. Lett. 100, 183001 (2008).

[11] A. N. Markevitch, D. A. Romanov, S. M. Smith, and R. J. Levis, Phys. Rev. Lett. 92, 063001 (2004).

[12] A. Hishikawa, A. Matsuda, M. Fushitani, and E. J. Takahashi, Phys. Rev. Lett. 99, 258302 (2007).

[13] A. N. Markevitch, D. A. Romanov, S. M. Smith, and R. J. Levis, Phys. Rev. Lett. 96, 163002 (2006).

[14] T. Ditmire, T. Donnelly, R. W. Falcone, and M. D. Perry, Phys. Rev. Lett. 75, 3122 (1995).

[15] A. McPherson, B. D. Thompson, A. B. Borisov, K. Boyer, and C. K. Rhodes, Nature (London) 370, 631 (1996).

[16] Y. L. Shao, T. Ditmire, J. W. G. Tisch, E. Springate, J. P. Marangos, and M. H. R. Hutchinson, Phys. Rev. Lett. 77, 3343 (1996).

[17] Z. Vager, R. Naaman, and E. P. Kanter, Science 244, 426 (1989).

[18] J. Levin, H. Feldman, A. Baer, D. Ben-Hamu, O. Heber, D. Zajfman, and Z. Vager, Phys. Rev. Lett. 81, 3347 (1998).
[19] F. Légaré, K. F. Lee, I. V. Litvinyuk, P. W. Dooley, A. D. Bandrauk, D. M. Villeneuve, and P. B. Corkum, Phys. Rev. A 72, 052717 (2005).

[20] C. Cornaggia, D. Normand, and J. Morellec, J. Phys. B 25, L415 (1992).

[21] C. Cornaggia, M. Schmidt, and D. Normand, Phys. Rev. A 51, 1431 (1995).

[22] S. Shimizu, J. Kou, S. Kawato, K. Shimizu, S. Sakabe, and N. Nakashima, Chem. Phys. Lett. 317, 609 (2000).

[23] S. Palaniyappan, R. Mitchell, N. Ekanayake, A. M. Watts, S. L. White, R. Sauer, L. E. Howard, M. Videtto, C. Mancuso, S. J. Wells, T. Stanev, B. L. Wen, M. F. Decamp, and B. C. Walker, Phys. Rev. A 82, 043433 (2010).

[24] T. Seideman, M. Y. Ivanov, and P. B. Corkum, Phys. Rev. Lett. 75, 2819 (1995).

[25] T. Zuo and A. D. Bandrauk, Phys. Rev. A 52, R2511 (1995).

[26] E. Lötstedt, T. Kato, and K. Yamanouchi, Phys. Rev. A 85, 041402 (2012).

[27] E. Runge and E. K. U. Gross, Phys. Rev. Lett. 52, 997 (1984).

[28] See Supplemental Material at http://link.aps.org/supplemental/ 10.1103/PhysRevA.86.043407 for an animation of the ionization and fragmentation dynamics.

[29] J. P. Perdew and A. Zunger, Phys. Rev. B 23, 5048 (1981).

[30] N. Troullier and J. L. Martins, Phys. Rev. B 43, 1993 (1991).

[31] K. Yabana, T. Nakatsukasa, J.-I. Iwata, and G. F. Bertsch, Phys. Status Solidi B 243, 1121 (2006).

[32] D. E. Manolopoulos, J. Chem. Phys. 117, 9552 (2002).

[33] I. Litvinyuk, K. Lee, P. Dooley, D. Rayner, D. Villeneuve, and P. Corkum, Phys. Rev. Lett. 90, 233003 (2003).

[34] V. Kumarappan, S. S. Viftrup, L. Holmegaard, C. Z. Bisgaard, and H. Stapelfeldt, Phys. Scr. 76, C63 (2007). 